\title{
Constraints on dark matter scattering with long-lived mediators using gamma-rays from the Sun
}

\author{
D. Serini ${ }^{* 1,2}$, F. Loparco ${ }^{1,2}$ and M. N. Mazziotta ${ }^{1}$ on behalf of the Fermi LAT \\ collaboration \\ ${ }^{1}$ Istituto Nazionale di Fisica Nucleare - Sezione di Bari, Italy \\ ${ }^{2}$ Dipartimento Interateneo di Fisica dell'Università e del Politecnico di Bari, Italy \\ E-mail: davide.serinilba.infn.it \\ E-mail: francesco.loparcolba.infn.it \\ E-mail: marionicola.mazziottaloba.infn.it
}

\begin{abstract}
The Large Area Telescope onboard the Fermi Gamma-Ray Space Telescope routinely observes the Sun during its data taking. In this work we selected a sample of gamma-ray events from the Sun and we have searched for possible features in their energy spectrum to search for dark matter signatures. The capture of dark matter particles in the Sun and their subsequent annihilation via long-lived dark mediators can produce gamma rays escaping from the Sun and reaching the Earth. This process is expected to yield a feature in the energy spectrum of gamma rays from the Sun, which depends on the scattering cross section of dark matter particles with solar nucleons. We do not find any statistically significant feature in the energy spectra and thus constrain both the spin-dependent and spin-independent scattering cross sections of dark matter particles with nucleons in a dark matter mass range from a few $\mathrm{GeV}$ up to a few tens of $\mathrm{GeV}$ at the level of $10^{-46}-10^{-45} \mathrm{~cm}^{2}$ and $10^{-48}-10^{-47} \mathrm{~cm}^{2}$, respectively.
\end{abstract}

36th International Cosmic Ray Conference -ICRC2019-

July 24th - August 1st, 2019

Madison, WI, U.S.A.

\footnotetext{
*Speaker.
} 


\section{Introduction}

One of challenges in modern experimental astrophysics is the indirect dark matter (DM) detection and the Sun is among the most promising targets for this task. DM particles coming from the Galactic halo could interact via elastic scattering with the nuclei in the solar envirovment. In such interaction, DM particles would lose energy and eventually be captured in the Sun core by its gravitational field. If these particles can also self-annihilate inside the Sun, an equilibrium between the capture and the annihilation mechanisms could be reached. The annihilation and decay products will include Standard Model (SM) particles, which can reach the Earth and be detected. All these particles should yield characteristic signals, whose strengths depend on the properties of the parent DM particles (i.e. mass and velocity), on the production process (i.e. DM annihilation/decay cross sections and branching ratios) and on the propagation from the Sun to the Earth.

Gamma rays are the most promising probes for indirect DM searches because, unlike charged particles, they are not deflected by the interstellar magnetic fields, thus making directional studies possible, and, unlike neutrinos, they are relatively easy to detect. However, gamma rays produced from DM annihilations in the Sun should be absorbed inside the Sun. In this work we investigate a scenario in which DM particles annihilate into intermediate light particles which are able to escape from the Sun and subsequently decay into gamma rays, which can therefore reach the Earth and be detected.

The Large Area Telescope (LAT) onboard the Fermi Gamma-Ray Space Telescope routinely observes the Sun during its data taking. In the present work we implemented a dedicated analysis based on a Poisson maximum likelihood approach to search for a possible DM signature in the energy spectrum of solar gamma rays. We use a 10-year dataset of gamma ray events collected by the Fermi-LAT in an energy range from $100 \mathrm{MeV}$ up to $150 \mathrm{GeV}$.

\section{Solar dark matter annihilation through a light intermediate state}

As mentioned above, gamma rays produced in the annihilations of DM particles in the Sun, as well as other SM particles with the exception of neutrinos, will be likely absorbed in the Sun. However, some authors [1-7] have proposed a possible scenario in which DM particles $\chi$ annihilate into long-lived scalar mediators $\phi$, which are able to escape from the Sun and then decay into SM particles, such as gamma rays or electron-positron pairs, that can be detected at Earth.

When equilibrium between the annihilation and capture processes is reached, the annihilation rate $\Gamma_{\text {ann }}$ is independent of the annihilation cross section and is set by the capture rate $\Gamma_{\text {cap }}$, which in turn depends on the scattering cross section (either spin-dependent, $\sigma_{\mathrm{SD}}$, or spin-independent, $\sigma_{\mathrm{SI}}$, on the local halo DM density number $\rho_{\mathrm{DM}}$, on the DM mass $m_{\chi}$, and on the DM velocity distribution:

$$
\Gamma_{\mathrm{ann}}=\frac{1}{2} \Gamma_{\mathrm{cap}}
$$

where the factor $\frac{1}{2}$ accounts for the two DM particles involved in each annihilation event [8-10].

In this scenario, DM particles are captured by the Sun through elastic scattering interactions with the solar nuclei. DM particles lose their energy in subsequent interactions until they sink into the core of the Sun, reaching thermal equilibrium. In the framework of this model, we assume that 
DM particles annihilate into a light intermediate state $\phi$, which subsequently decays into photons through the processes $\chi \chi \rightarrow \phi \phi$ and $\phi \rightarrow \gamma \gamma$. In this picture we implicitly assume that the mediators $\phi$ are able to escape outside the Sun without further interactions and that, after escaping from the Sun, each $\phi$ decays producing a pair of gamma rays. If this happens, the gamma rays produced can reach the Earth and may be detectable as a signature of DM in the form of an excess of photons from the Sun direction.

The DM particles are assumed to annihilate at rest in the Sun core, and in the lab frame the energy of the mediator $\phi$ will be equal to the mass $m_{\chi}$ of the DM particle, i.e. $E_{\phi}=m_{\chi}$. If we also assume that the $\phi$ is a light scalar such that $m_{\phi} \ll m_{\chi}$, the angular dispersion of the daughter gamma rays with respect to the direction of the parent $\phi$ will be negligible. Under these assumption, indicating with $R_{\odot}$ the solar radius, $D$ the Sun-Earth distance and $L$ the $\phi$ decay length; the DM gammaray flux at Earth $\Phi_{\mathrm{DM}}(E)$ will be equivalent to that from a point-like source centered in the Sun's core $\left(\Gamma_{\text {cap }} / 4 \pi D^{2}\right)$, modulated by the survival probability of the mediator $\left(e^{-R_{\odot} / L}-e^{-D / L}\right)[8]$ :

$$
\Phi_{\mathrm{DM}}(E)=N_{\gamma}(E) \frac{\Gamma_{\text {cap }}}{4 \pi D^{2}}\left(e^{-R_{\odot} / L}-e^{-D / L}\right)
$$

Under the assumptions of this model, the resulting DM photon spectrum will have a box-shape, where the center and the width of the box will depend on $m_{\chi}$ and $m_{\phi}$ [12]. In the hypothesis of a light mediator, the dependence on $m_{\phi}$ is lost and the box extends from $E=0$ to $E=m_{\chi}$. In this case $N_{\gamma}(E)$ can be written as $N_{\gamma}(E)=2 H\left(m_{\chi}-E\right) / m_{\chi}$, where $H$ is the Heaviside step function and the factor 2 takes into account the fact that for each mediator decay two photons are produced.

\section{Data analysis}

The Sun is visible in gamma rays because of the interaction of cosmic rays (CRs) with the solar environment, and the standard solar emission mechanisms are expected to yield a smooth gammaray spectrum. The DM scenario illustrated above results in a hard spectrum, which is expected to yield a characteristic feature in the solar gamma-ray energy spectrum, which we search for in this work. We implemented a Poisson maximum likelihood fitting procedure in order to search for possible local excesses in the count spectra of photons from the signal region. Our analysis approach is based on an ON/OFF technique, in which we combine the data from a Region of Interest (RoI) of $2^{\circ}$ angular radius centered on the position of the Sun (hereafter "signal region" or "ON region") and from a RoI of the same size centered on the anti-Sun (hereafter "background region" or "OFF region") ${ }^{1}$. With this approach, possible systematic effects are automatically included in the analysis, since they should appear in both the "ON" and "OFF" regions.

The fits are performed in sliding energy windows to search for DM signatures, which should yield local excess counts on the top of a smooth spectrum. The energy range scanned extends from $100 \mathrm{MeV}$ to $150 \mathrm{GeV}$ and is divided in 64 bins per decade, equally spaced on a logarithmic scale. Each energy window is defined as the interval $\left[(1-w) E_{\mathrm{w}},(1+w) E_{\mathrm{w}}\right]$, where $E_{\mathrm{w}}$ is the energy corresponding to the center of the window and $w E_{\mathrm{w}}$ is the half-width of the window. The

\footnotetext{
${ }^{1}$ At any given time, the position of the anti-Sun is defined by the Sun position with a 6 months offset. In this way the background and the signal regions will span the same portion of sky, the exposures of the two regions will be nearly equal and the anti-Sun will be always separated of about $180^{\circ}$ from the Sun
} 
excesses in the count spectrum are assumed to be originated from a box-like feature in the energy spectrum [8-11]. When folded with the instrument energy response function, this feature should appear as a smooth edge, with the smoothing originated from the energy resolution of the LAT. For this reason, the parameter $w$ is chosen in order to ensure that the width of the windows is larger than the LAT energy resolution for the whole energy range explored in the analysis. For the present analysis we choose to use a window size of $\mathrm{w}=0.6$.

In Figure 1 the observed count distributions in the "ON" and "OFF" regions are shown respectively in black and blue. The excess of counts in the signal region is due to the steady solar gamma-ray emission.

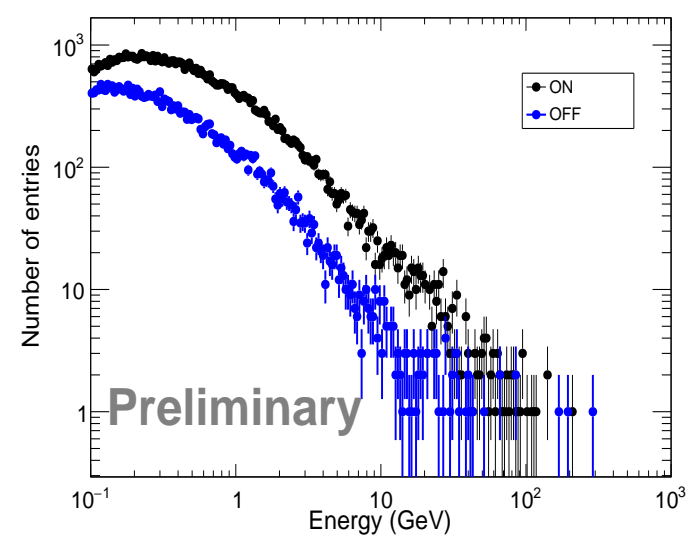

Figure 1: Observed count distributions in the "ON" and "OFF" regions. Both regions have an angular radius of $2^{\circ}$. The energy interval has been divided in 64 bins per decade.

The expected counts $\mu_{i}^{\text {ON/OFF }}$ in the $i$-th bin of reconstructed energy in the two regions can be written as:

$$
\begin{aligned}
\mu_{\mathrm{i}}^{O N} & =\int d E_{\mathrm{t}} \mathscr{E}_{O N}\left(E_{i} \mid E_{\mathrm{t}}\right) \cdot\left[\Phi_{\mathrm{sig}}\left(E_{\mathrm{t}}\right)+\Phi_{b k g}\left(E_{\mathrm{t}}\right)\right] \\
\mu_{\mathrm{i}}^{O F F} & =\int d E_{\mathrm{t}} \mathscr{E}_{O F F}\left(E_{\mathrm{i}} \mid E_{\mathrm{t}}\right) \cdot \Phi_{\mathrm{bkg}}\left(E_{\mathrm{t}}\right)
\end{aligned}
$$

where $E_{\mathrm{t}}$ is the true photon energy, $\mathscr{E}_{\mathrm{ON} / \mathrm{OFF}}\left(E_{\mathrm{i}} \mid E_{\mathrm{t}}\right)$ is the exposure evaluated by means of the Monte Carlo simulations taking into account the livetime distributions as a function of the off-axis angle in the instrument frame, $\Phi_{\text {sig }}\left(E_{\mathrm{t}}\right)$ is the net signal flux from the Sun, which contributes only to the counts in the "ON" region, and $\Phi_{\mathrm{bkg}}\left(E_{\mathrm{t}}\right)$ is the background flux (diffuse, point sources, irreducible background), which contributes to the counts in both the "ON" and "OFF" regions.

Each flux contribution can be expressed as the sum of a continuous smooth component $\Phi_{0}(E)$ with a possible additional feature $\Phi_{\mathrm{f}}(E)$. Since the energy windows are narrow, the continuous term $\Phi_{0}(E)$ can be well described as a simple power law model (PL) $\Phi_{0}(E \mid k, \alpha)=k\left(E / E_{0}\right)^{-\alpha}$, where $\alpha$ is the spectral index and the prefactor $k$ (in units of $\mathrm{MeV}^{-1} \mathrm{~cm}^{-2} \mathrm{~s}^{-1}$ ) corresponds to the photon flux at the scale energy $E_{0}$, fixed at $100 \mathrm{MeV}$. For the spectral feature, we assume a box-like (box) feature $\Phi_{\mathrm{f}}(E \mid s)=s H\left(E_{\mathrm{w}}-E\right)$, where $E_{\mathrm{w}}$ is the energy corresponding to the center of the window, $H$ is the Heaviside step function and $s$ represents the intensity of the feature. 
To test the significativity of a signal feature we evaluate the local Test Statistic $T S=2 \Delta \log \mathscr{L}=$ $2\left(\log \mathscr{L}_{1}-\log \mathscr{L}_{0}\right)$, where $\mathscr{L}_{0}$ and $\mathscr{L}_{1}$ are the values of the likelihood functions obtained when fitting the data with the models corresponding to the null hypothesis (i.e. no feature included) and the alternative hypothesis (i.e. feature included) respectively. Since the two models are nested and the null hypothesis model can be obtained from the alternative hypothesis model setting $s_{\text {sig }}=0$, we expect that $T S$ should obey a $\chi^{2}$ distribution with one degree of freedom.

\section{Results}

The plot on the left in Figure 2 shows the upper limits (ULs) al 95\% confidence level on the intensity of the box-like feature predicted by the light mediator model (see sec. 2). The plot also shows the confidence belts, which have been evaluated with the pseudo-experiment technique. To evaluate the expectation bands for our results, i.e. the sensitivity to the null hypothesis, we have chosen a smoothly broken power-law fit to our data [8-11] as our template and performed 1000 pseudo-experiments starting from this template spectrum. The containment bands for the parameters and the $T S$ values are then evaluated as the quantiles of the fitted distributions. It is possible to observe that the fitted parameters lie within the central $95 \%$ confidence belt in all the energy windows.

The other plots in Figure 2 show the local significance (center plot) and the global significance (right plot) of the possible feature at different energies for the model considered in this work. We note that the $T S_{\text {local }}$ values exhibit a peak in the energy windows around $E=500 \mathrm{MeV}$. This is due to a change in the slope of the observed count distribution (see Fig.1) that is interpreted by our fitting algorithm as a "feature" in the spectrum. Nevertheless we see that in nearly all energy windows the values of $T S_{\text {local }}$ lie within the $95 \%$ expectation band.To convert the $T S_{\text {local }}$ into a global significance we used the results of the 1000 pseudo-experiments ${ }^{2}$. For each pseudo-experiment, we record the largest value of the local Test Statistic $T S_{\max }$; then, we evaluate the quantiles of the distribution of $T S_{\max }$, and the corresponding values of the global significance $s_{\text {global }}$, assuming that it obeys to a half-normal distribution. The results after the global significance conversion are
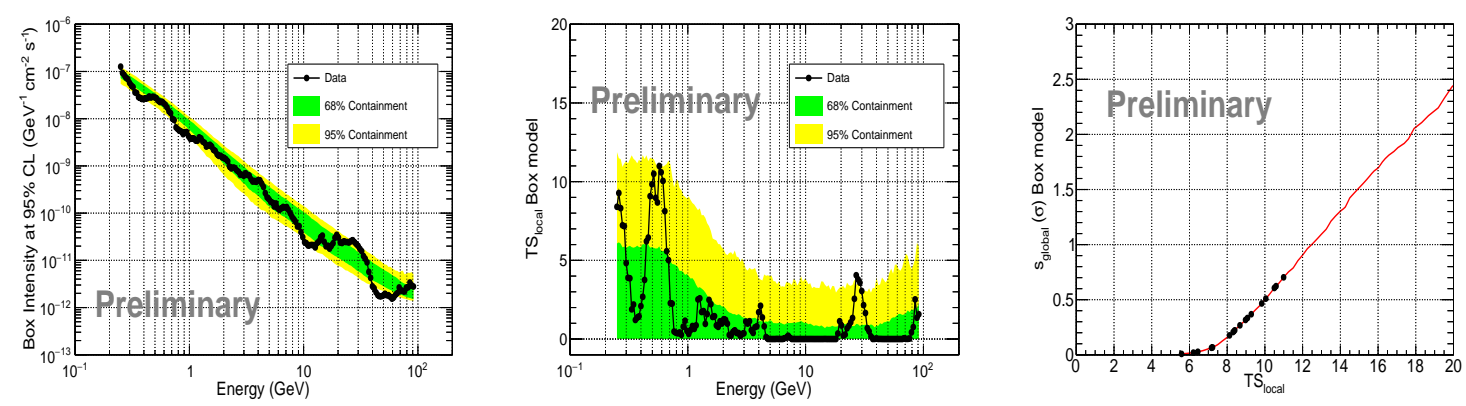

Figure 2: Upper limit at 95\% confidence level (left panel), Local TS (center panel) and global significance (right panel) of the feature intensity in the solar solar gamma-ray spectrum in the light mediator scenario (box model). The red lines in the right plot indicate the conversion from $T S_{\text {local }}$ to $s_{\text {global }}$, while the black dots correspond the most significant features.

\footnotetext{
${ }^{2}$ Each pseudo experiment corresponds to a simulation of one full search across the whole energy range.
} 

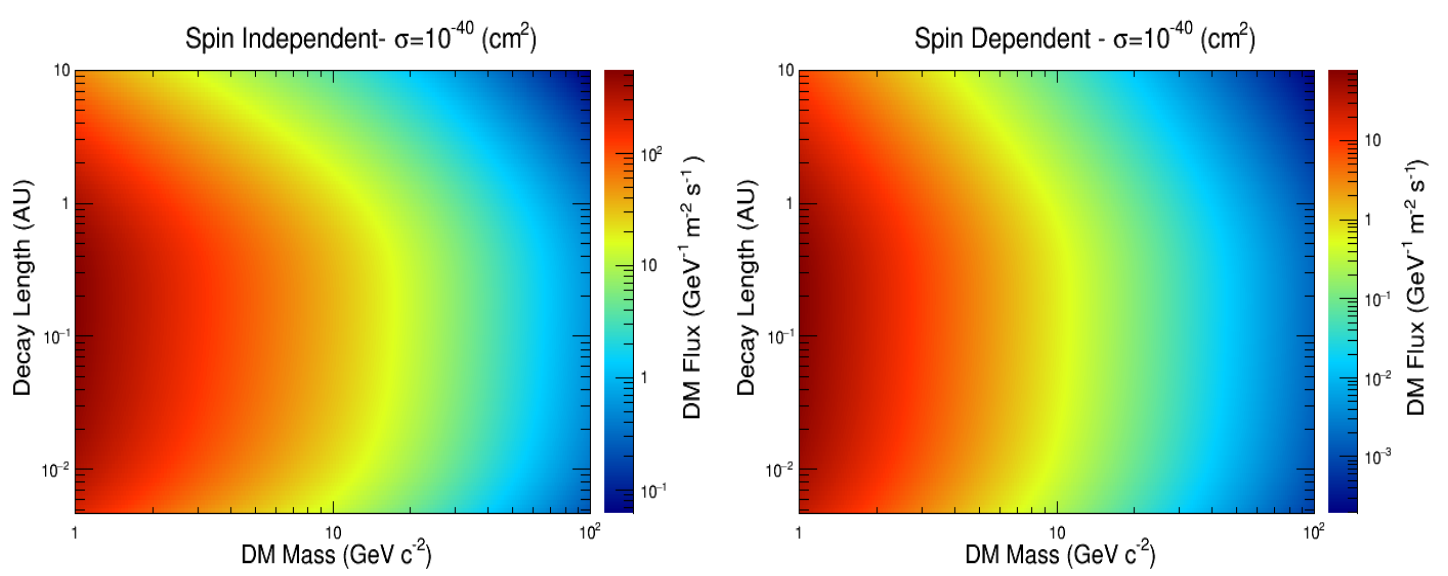

Figure 3: Expected gamma-ray flux at the Earth from DM annihilations in the Sun via long-lived mediators as a function of the DM mass and decay length, for the spin independent (top panel) and spin dependent (bottom panel). In both cases a scattering cross section of $10^{-40} \mathrm{~cm}^{2}$ is assumed.

shown in the right plots in Fig.2. The most significant features have global significances less than or comparable to $1 \sigma$ in both cases, and therefore are globally insignificant.

\section{Constraints on DM-nucleon cross section with long-lived mediators}

Using Eq.2.2, the upper limits on the flux can be converted into limits on the DM-nucleon cross section.

The capture rate $\Gamma_{\text {cap }}$ has been calculated with the DARKSUSY code version 6.1 .0 [13-15] assuming the default settings, i.e. a local DM density $\rho_{\odot}=0.3 \mathrm{GeV} / \mathrm{cm}^{3}$, a DM-nucleon scattering cross section $\sigma=10^{-40} \mathrm{~cm}^{2}$ (in both the spin-dependent and spin-independent cases) and a Maxwellian velocity distribution for DM particles with average $v_{\odot}=220 \mathrm{~km} / \mathrm{s}$ and dispersion $v_{r m s}=270 \mathrm{~km} / \mathrm{s}$. A change in these values will result in a rescaling of the total capture rate Fig.3 shows the expected gamma-ray fluxes at Earth from DM annihilations in the Sun core via long-lived mediators as a function of the DM mass and decay length.

Using the values of $\Phi_{\mathrm{DM}}(E)$ calculated with the DARKSUSY code (Fig. 3), we evaluated the limits on the DM-nucleon cross section as:

$$
\sigma_{\mathrm{UL}}\left(m_{\chi}\right)=\frac{\Phi_{\mathrm{UL}}\left(E=m_{\chi}\right)}{\Phi_{\mathrm{DM}}\left(E=m_{\chi}\right)} \times 10^{-40} \mathrm{~cm}^{2} .
$$

The value of $10^{-40} \mathrm{~cm}^{2}$ in the previous equation corresponds to the value of the DM-nucleon cross section used to calculate the capture rates shown in Figure 3.

The left panel in Figure 4 shows the constraints on DM annihilation to $\gamma \gamma$ via an intermediate state, obtained from the upper limits on the intensity of the box feature, assuming that DM capture takes place either via spin-independent scattering (black lines) or spin-dependent scattering (red lines). The constraints have been calculated for four values of the decay length of the intermediate state, $L=R_{\odot}, 0.1,1$ and $5 \mathrm{AU}$. The limits on the cross-sections evaluated in this work are in the range from about $10^{-46}$ to $10^{-45} \mathrm{~cm}^{2}$ for the spin-dependent cross section and in the range from about $10^{-48}$ to $10^{-47} \mathrm{~cm}^{2}$ for the spin-independent cross section. The ranges of variation depend 

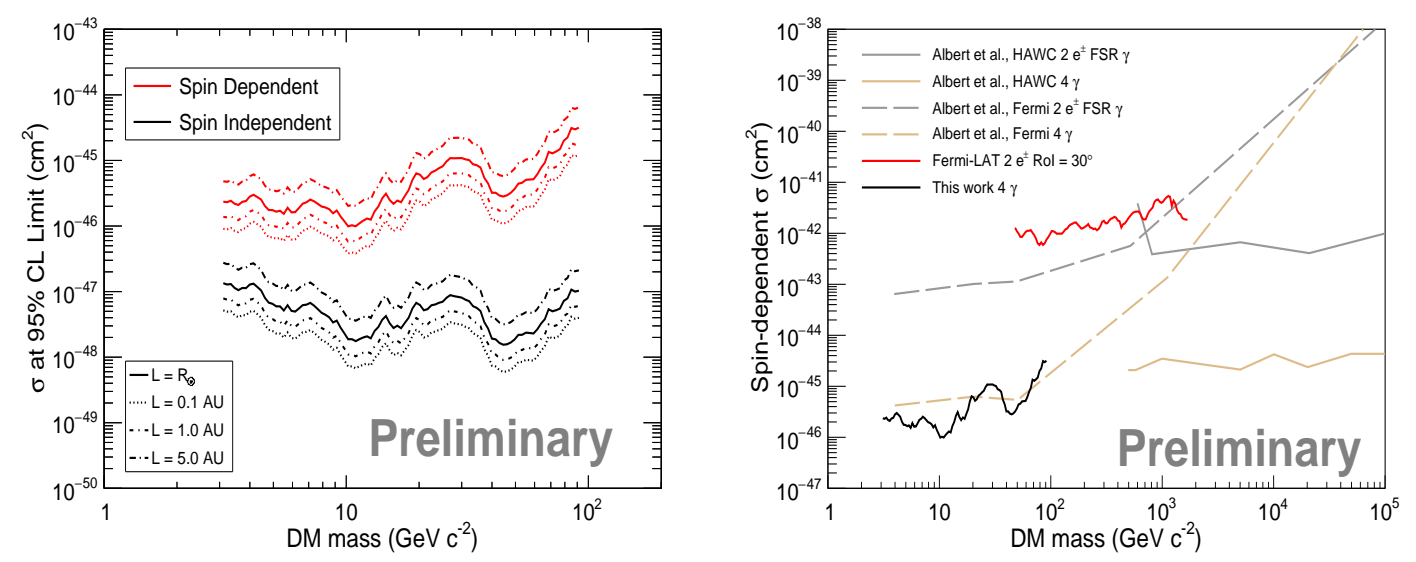

Figure 4: On the left: limits on the DM-nucleon cross section via long-lived mediator for four different decay lengths $L=R_{\odot}, 0.1,1$ and $5 \mathrm{AU}$ obtained in this work. On the right: Upper limits on the spindependent DM-nucleon scattering cross section for the long lived mediator with decay length $L=R_{\odot}$. Our results (black line) are shown together with those from the HAWC and Fermi [16] with gamma rays from DM annihilating into $2 e^{ \pm}$, i.e., $2 e^{ \pm}$FSR $\gamma$ case, (gray lines) and into $4 \gamma$ (brown lines), and electron/positron Fermi-LAT results (red line) [8-10].

on the life time of the mediator considered. We do not find any statistically significant feature in the energy spectra and thus constrain both the spin-dependent and spin-independent scattering cross sections of dark matter particles with nucleons in a dark matter mass range from a few $\mathrm{GeV}$ up to a few tens of $\mathrm{GeV}$ at the level of $10^{-46}-10^{-45} \mathrm{~cm}^{2}$ and $10^{-48}-10^{-47} \mathrm{~cm}^{2}$, respectively. The model considered in this work is the same as investigated by other authors in their recent works $[4,16]$. A summary of the limits on the spin-dependent DM-nucleon cross section for the long-lived mediator scenario with decay length $L=R_{\odot}$ is shown in the plot on the right in Fig. 4, together with the recent constraints obtained from the analysis of solar gamma rays by HAWC and Fermi [16] for the dark matter scenarios discussed, in which the mediator decays into 2 photons ( $4 \gamma$ case), and for the case in which the mediator decays into $e^{ \pm}$pairs and photons are produced as final state radiation ( $2 e^{ \pm}$FSR $\gamma$ case) $[8-10]$.

\section{Conclusion}

In this work we implemented a dedicated analysis based on a Poisson maximum likelihood fitting approach in order to search for possible dark matter features in the energy spectrum of gamma rays coming from the Sun using a 10-year Fermi-LAT dataset. We considered a scenario in which gamma rays are produced in the decay of long-lived mediators produced via the annihilations of DM particles captured in the Sun.

We do not find any statistically significant feature in the energy spectra and thus constrain both the spin-dependent and spin-independent scattering cross sections of dark matter particles with nucleons in a dark matter mass range from a few $\mathrm{GeV}$ up to a few tens of $\mathrm{GeV}$ at the level of $10^{-46}-10^{-45} \mathrm{~cm}^{2}$ and $10^{-48}-10^{-47} \mathrm{~cm}^{2}$, respectively.

The limits obtained in this work are similar to those from the HAWC and Fermi collaboration in in [16] (see Fig. 4), where limits on the spin dependent cross-section were determined on dark 
matter particle masses in the range from $4 \mathrm{GeV}$ to $10^{6} \mathrm{GeV}$ by combining Fermi-LAT and 3-years of HAWC observations of the Sun and complete them in the lower mass $(<100 \mathrm{GeV})$ range.

\section{Acknowledgments}

The Fermi-LAT Collaboration acknowledges support for LAT development, operation and data analysis from NASA and DOE (United States), CEA/Irfu and IN2P3/CNRS (France), ASI and INFN (Italy), MEXT, KEK, and JAXA (Japan), and the K.A. Wallenberg Foundation, the Swedish Research Council and the National Space Board (Sweden). Science analysis support in the operations phase from INAF (Italy) and CNES (France) is also gratefully acknowledged. This work performed in part under DOE Contract DE-AC02-76SF00515.

\section{References}

[1] M. Pospelov, A. Ritz, and M. B. Voloshin. Phys. Lett., B662 53-61, 2008.

[2] N .A. Hamed, D. P. Finkbeiner, T. R. Slatyer, and N. Weiner. Phys. Rev., D79 015014, 2009.

[3] P. Schuster, N. Toro, N. Weiner, and I. Yavin. Phys. Rev., D82 115012, 2010.

[4] R. K. Leane, K. C. Y. Ng, and J. F. Beacom. Phys. Rev., D95(12) 123016, 2017.

[5] P. Schuster, N. Toro, and I. Yavin. Phys. Rev., D81 016002, 2010.

[6] N. F. Bell and K. Petraki. JCAP, 1104 003, 2011.

[7] C. Arina, M. Backovic, J. Heisig, and M. Lucente. Phys. Rev., D96(6) 063010, 2017.

[8] M. N. Mazziotta, F. Loparco, A. Cuoco, P. De La Torre Luque, F Gargano, M. Gustafsson and D. Serini, [Fermi-LAT Collaboration]. A search for dark matter cosmic-ray electrons from the Sun with the Fermi Large Area Telescope. To be submitted, 2019.

[9] M. N. Mazziotta, F. Loparco, D. Serini, A. Cuoco, P. De La Torre Luque and F Gargano, [Fermi-LAT Collaboration]. Search for dark matter signature in the gamma ray towards the Sun with the Fermi Large Area Telescope. To be submitted, 2019.

[10] M. N. Mazziotta, A. Cuoco, P. De La Torre Luque, F. Loparco and D. Serini, [Fermi-LAT Collaboration]. In proceeding of 36th ICRC POS ( ICRC2019) 5312019

[11] M. N. Mazziotta, F. Costanza, A. Cuoco, F. Gargano, F. Loparco and S. Zimmer, [Fermi-LAT Collaboration]. Phys. Rev. D 98022006 (2018) [arXiv:1712.07005].

[12] A. Ibarra, S. L. Gehler, and M. Pato. JCAP, 1207 043, 2012.

[13] P. Gondolo, J. Edsjo, P. Ullio, L. Bergstrom, M. Schelke, and E. A. Baltz. JCAP, 0407 008, 2004.

[14] T. Bringmann, J. Edsja, P. Gondolo, P. Ullio, and L. Bergstrom. JCAP, 1807(07) 033, 2018.

[15] J. Edsjo, T. Bringmann, P. Gondolo, , P. Ullio, L. Bergstrom, M. Schelke, E. A. Baltz, and G. Duda. http://www.darksusy.org/.

[16] A. Albert et al. Phys. Rev. D, 2018. [Phys. Rev.D98 123012 (2018)]. 